This is the final peer-reviewed accepted manuscript of:

Luca De Marchi, Alessandro Marzani, Jochen Moll, Paweł Kudela, Maciej Radzieński, Wiesław Ostachowicz, A pulse coding and decoding strategy to perform Lamb wave inspections using simultaneously multiple actuators, Mechanical Systems and Signal Processing, Volume 91, 2017, Pages 111-121, ISSN 0888-3270

The final published version is available online at:

https://doi.org/10.1016/j.ymssp.2016.12.014

Rights / License:

The terms and conditions for the reuse of this version of the manuscript are specified in the publishing policy. For all terms of use and more information see the publisher's website.

This item was downloaded from IRIS Università di Bologna (https://cris.unibo.it/)

When citing, please refer to the published version. 


\title{
A pulse coding and decoding strategy to perform Lamb wave inspections using simultaneously multiple actuators
}

\author{
Luca De Marchi ${ }^{1,1}$, Alessandro Marzani ${ }^{1, b}, J^{\prime}$ Jochen Moll ${ }^{1, \mathrm{c}, *}$, Paweł Kudela ${ }^{1, \mathrm{~d}}$, Maciej Radzieński ${ }^{1, \mathrm{~d}}$, \\ Wiesław Ostachowicz ${ }^{1, \mathrm{~d}}$ \\ ${ }^{a}$ Department of Electrical, Electronic, and Information Engineering, University of Bologna, Italy \\ ${ }^{b}$ Department of Civil, Environmental and Materials Engineering - DICAM, University of Bologna, Italy \\ ${ }^{c}$ Department of Physics Soethe University of Frankfurt am Main, Germany \\ ${ }^{d}$ Institute of Fluid Flow Machinery, Polish Academy of Sciences, Poland
}

\begin{abstract}
The performance of Lamb wave based monitoring systems, both in terms of diagnosis time and data complexity, can be enhanced by increasing the number of transducers used to actuate simultaneously the guided waves in the inspected medium. However, in case of multiple simultaneously-operated actuators the interference among the excited wave modessignals within the acquired signals has to be considered for the further processing. To this aim, in this work a code division strategy based on the Warped Frequency Transform is presented. At first, the proposed procedure encodes actuation pulses using Gold sequences. Next, for each considered actuator the acquired signals are compensated from dispersion by cross correlating the warped version of the actuated and received signals. Compensated signals form the base for a final wavenumber imaging meant at emphasizing defects and or anomalies by removing incident wavefield and edge reflections. The proposed strategy is tested numerically, and validated through an experiment in which guided waves are actuated in a plate by four piezoelectric transducers operating simultaneously.
\end{abstract}

Keywords: Lamb wave inspections, Spread spectrum pulse coding, Warped Frequency Transform, Wavenumber filtering, Laser vibrometry

\section{Introduction}

In recent years, ultrasonic Guided Waves (GW) received a considerable attention from the nondestructive evaluation (NDE) and structural health monitoring (SHM) community because of the GW ability to travel long distances without substantial attenuation as well as to their versatile multimode/frequency nature that allows for defect classification and sizing [1]. Among the main applications, the detection and localization of defects and impact in plate-like components has gathered the attention of several research fields including aeronautics. In this context, GW are usually excited and sensed through multiple piezo-electric transducers arranged in different array configurations. The receivers can be triggered on the actuator and the acquired time-waveforms are subsequently analysed to locate and characterize defects and/or impacts.

*corresponding author: phone +49 (0) 69 798-47208, fax +49 (0) 69 798-47221

Email addresses: 1.demarchi@unibo.it (Luca De Marchi), alessandro.marzani@unibo.it (Alessandro Marzani), moll@physik.uni-frankfurt.de (Jochen Moll ), pk@imp.gda.pl> (Paweł Kudela), mradzienski@imp.gda.pl] (Maciej Radzieński ), wieslaw@imp.gda.pl (Wiesław Ostachowicz ) 
Similarly, piezo-actuators can be combined with non-contact receivers, such as Scanning Laser Doppler Vibrometers (SLDV) [2], or air coupled probes [3].

In order to enhance the inspection abilities of such monitoring systems, it can be convenient to increase the number of transducers (probes) used for the monitoring task. Multiprobe systems can be implemented in several different configurations and can be distinguished on the basis of the level of coordination among the probes [4]. One common configuration in GW inspections is based on the use of a "single TX - multiple RX", that is the use of a single transmitting transducer (TX) and multiple receiving transducers (RXs), i.e. one actuator and multiple sensors. This solution has a clear advantage with respect to "single TX - single RX" systems because it allows for an $N_{R}$-fold reduction of the acquisition time, being $N_{R}$ the number of receivers from which the signal is gathered. However, there are still some limitations in operating "single TX - multiple RX". Firstly, devices such as SLDVs or air coupled probes acquire single-point measurements sequentially. This means that to acquire multiple scan-points simultaneously the acquisition devices should be replicated. This is very unpractical given the high costs of such equipments and unfeasible if the number of desired RX grows. Secondly, the usage of a single transmitting transducer might bring some risk, in the sense that if the defect cross-section is small and it is located perpendicularly to the wavefronteross-section along the actuated wave directions of propagation is small, most likely the defect will not be detected.

As such, multiple transmitting transducers procedures, i.e. "multiple TX", are generally adopted to reduce such risks. Conventional multiple TX systems for guided wave based structural health monitoring usually relies on a time-division multiplexing scheme, where each piezoelectric transducer acts in turn as the transmitter. In this context, either tone-burst signals [5] or chirp signals [6] are widely used. This solution is very effective but, if $N_{T}$ is the number of TXs, the total inspection time is a linear function of the product $N_{T} \times N_{R}$.

An alternative solution is to operate simultaneously multiple TXs. The presence of multiple simultaneously operated TXs makes the system radically different from the other considered cases, introducing the possibility of interference among the multiple excitations injected in the system within the received signals.

To treat such interference, one may use frequency division [7]. However, frequency division limits the successful usage of pulse-compression because it hampers the excitation of large bandwidth pulses which are required to achieve high resolution defect imaging. In addition, frequency division suffers the dispersive and multi-modal nature of guided waves due to which, for the same distance of propagation, the same wave attenuates differently at different frequencies.

Another possible alternative is given by the use of code division [8]. Such alternative has a clear advantage in terms of transducer bandwidth exploitation, but it requires some special processing when applied to systems based on GWs. In fact it is mandatory to counteract the detrimental effect of dispersion and multi-modal propagation to achieve the desired pulse-compression performances. To this aim, a pulse coding and decoding strategy based on the Warped Frequency transform (WFT) [9] is proposed in this work. The WFT has two beneficial effects: 1) it compensates the acquired signals for dispersion, 2) it preserves the pseudo-orthogonality of the excited waveforms, because it is computed with a unitary operator, thus allowing, at the same time, efficient pulse compression and interference cancellation. A subsequent postprocessing procedure based on wavenumber filtering [10] is applied to the pulse compressed signals for the final damage imaging.

The paper is organized as follows: in section 2 pulse encoding via Gold codes will be reviewed and its limitation for distance estimation purposes in guided waves based approaches is highlighted. In section 3 the proposed dispersion compensation and pulse compression procedures will be presented and applied to some numerically simulated waveforms. The advantages of the proposed strategy are shown through an experimental validation aimed at detecting a notch in an aluminium plate in section 4 . The conclusions end 
the paper.

\section{Spread spectrum pulse coding}

In the bulk ultrasound research field, several schemes that allows to operate "multiple-TX" systems by using encoded pulses have been successfully presented [11]. In this paper, the employed encoding is based on Gold-codes which provide excellent cross-correlation properties with minimum mutual interference [12]. This methodology removes the requirement of a switching network, because demodulation can be achieved in post-processing by means of a matched filter (cross-correlation function), and this is the reason for their large usage in wireless CDMA-communication systems since they can provide multi-user access.

In Fig. 1(a) and (b) two sample $T x_{i}$ signals are depicted. The two signals are obtained considering two different Gold sequences of length $L=2^{5}-1$ applied to a sinusoid of frequency of $200 \mathrm{kHz}$. The autocorrelation of $T x_{1}$ (in Fig. 1(a)) and the cross-correlation between $T x_{1}$ and $T x_{2}$ are plotted in Fig 1(c). From Fig. 1(c) it can be seen that the autocorrelation is very peaky and that the cross-correlation between different coded pulses is very low. Such properties imply that such kind of pulses are very well suited for time-offlight estimation problems when the acquired signals are corrupted by jamming interferences produced by multiple simultaneously actuated pulses as in "multiple-TX" systems. Other coding strategies have been successfully applied to ultrasound inspections [13], however the benefit brought by coded waveforms are almost completely lost when adopted for GW inspections, in case of highly dispersive propagation.

In Fig. 2 it can be seen how the decoding works when applied to a numerically simulated guided waves signal $s(t, D)$ represented in Fig. 2(b). In particular, the signal has been computed by simulating the propagation of Lamb waves in a $3 \mathrm{~mm}$ thick aluminium plate using Fourier domain techniques [14]. A propagation distance of $D=200 \mathrm{~mm}$ between the transmitter (TX) and the receiver (RX) has been used. As actuation pulse $s(t, 0)$ the Gold modulated sinusoid of Fig. 2(a) centered at $200 \mathrm{kHz}$ with Gold codes of length $L=2^{5}-1$ has been employed. More specifically, $s(t, D)$ was computed by modeling the considered "single TX - single RX" system in the frequency domain as an undamped dispersive system whose response is

$$
S(f, D)=S(f, 0) \cdot e^{-j 2 \pi \int_{0}^{f} \tau_{D}^{n}(\alpha) d \alpha}
$$

In Eq. (1) $S(f, 0)=\mathbf{F}\{s(t, 0)\}$ is the Fourier transform of the encoded actuation pulse, being $\mathbf{F}$ the Fourier operator, and $\tau_{D}^{n}(f)=\frac{D}{c_{g}^{n}(f)}$ is the group delay of the $n-t h$ guided wave at frequency $f$ computed as the ratio between the distance of propagation $D$ and the wave group speed at the same frequency $c_{g}^{n}(f)$. Finally, the inverse Fourier transform of $S(f, D)$ leads to the simulated signal $s(t, D)$. In this example, it was assumed that the transducer excites only the $A_{0}$ Lamb wave mode and the group delay curve for such mode was obtained by using the Semi-Analytical Finite Element (SAFE) formulation proposed in [15].

Due to dispersion, the received waveform $s(t, D)$ is considerably distorted, as can be seen in Fig. 2 (b). The cross-correlation between the actuated pulse and the received signal is represented in Fig 2(c) where the distances on the $x$-axis have been obtained by multiplying the temporal lags to the phase velocity of the $A_{0}$ at $200 \mathrm{kHz}$ (matched filter response) to estimate the distances traveled by the wave frequency components. As can be seen from Fig. 2(c) the detrimental effect of dispersion precludes the possibility of having a good pulse compression as well as to extrapolate a good estimation of the traveled distance $D$. Hence, in the following section a dispersion compensation procedure, based on the Warped Frequency transform (WFT) is included in the processing algorithm.

Fig. 3 illustrates the difference between conventional toneburst excitation and binary phase shift keying (BPSK) waveforms. An aluminum plate with a thickness of $1.5 \mathrm{~mm}$ at a carrier frequency of $70 \mathrm{kHz}$ is 
(a)

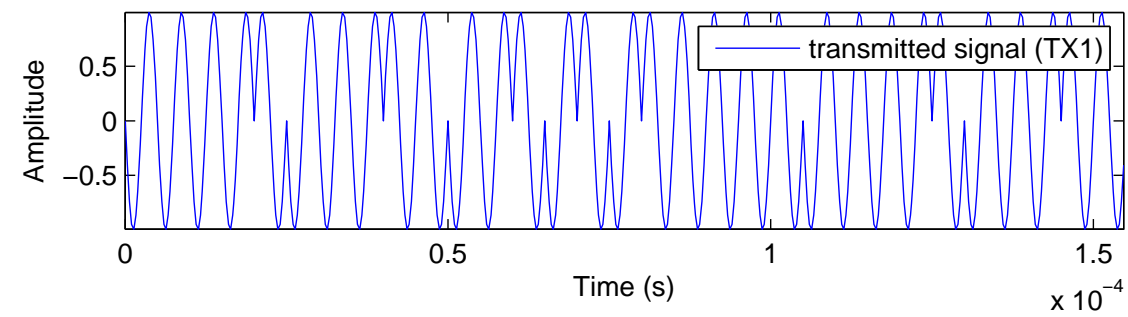

(b)
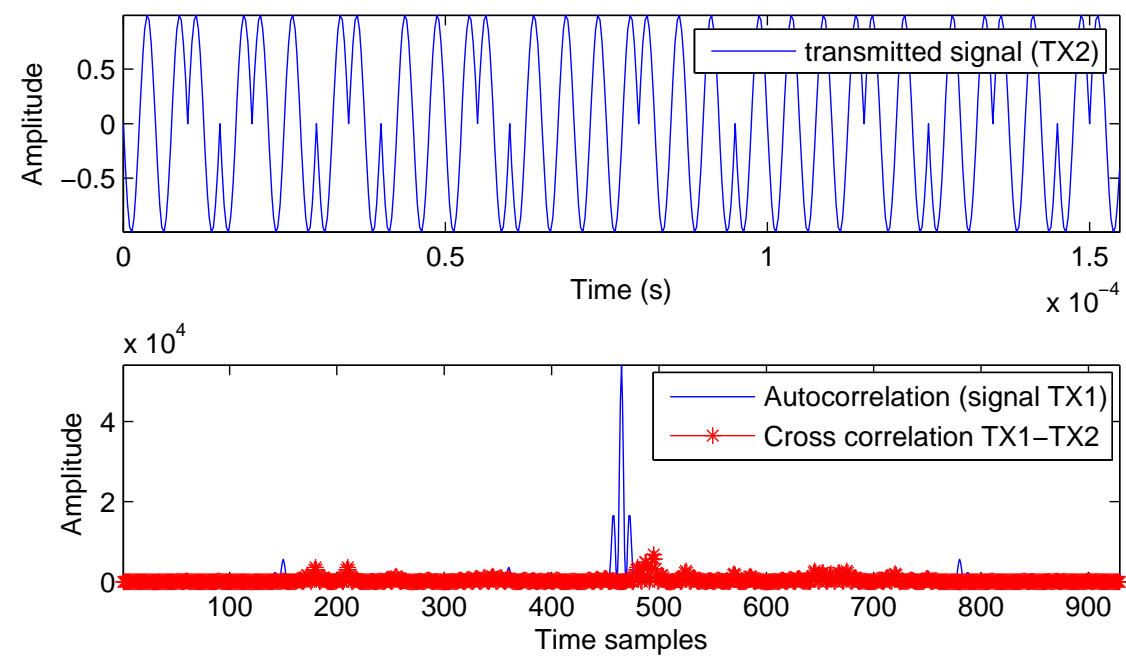

Figure 1: (a-b) two spread spectrum pulses based on Gold sequences of length $N=2^{5}-1$; (c) autocorrelation of the pulse TX1 and cross-correlation between pulses TX1 and TX2.

considered. In this analytical example the S0-mode wave motion is studied where dispersion does not play a significant role. In both cases the correct propagation distance of $0.5 \mathrm{~m}$ is detected in the matched filter response, but the waveforms differ in terms of waveform extend as well as the sidelobes in the case of BPSK-signals..

\section{Damage imaging for spread spectrum coded pulses}

\subsection{Lamb waves dispersion compensation}

The Warped Frequency transform (WFT) has been used as an effective tool to compensate guided waves signal for dispersion [16]. In particular, given a generic signal $s(t)$ whose frequency representation is $S(f)=$ $\mathbf{F}\{s(t)\}$, the Frequency Warping operator $\mathbf{W}_{w}$ reshapes the periodic frequency axis by means of a proper function $w(f)$, that we will call from now on warping map, such as:

$$
\begin{aligned}
s_{w}(t) & =\mathbf{W}_{w}\{s(t)\} \\
S_{w}(f)=\mathbf{F}\left\{s_{w}(t)\right\} & =\sqrt{\dot{w}(f)} \cdot S(w(f))
\end{aligned}
$$


(a)

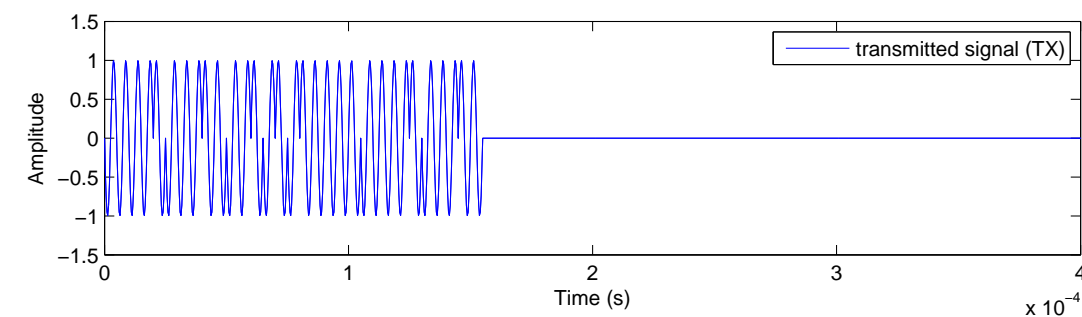

(b)

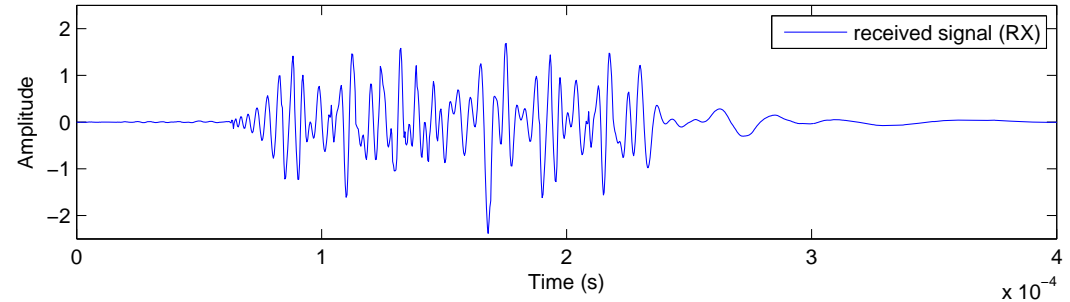

(c)

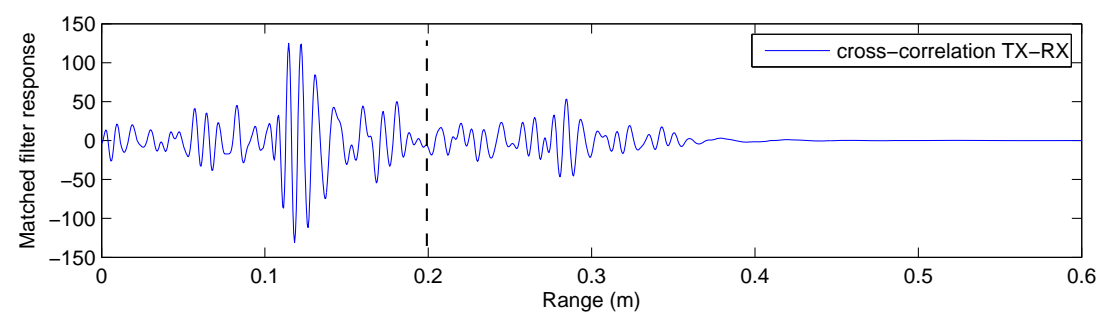

Figure 2: (a) actuation sinusoidal pulse $s(t, 0)$ centered at $200 \mathrm{kHz}$ and modulated by Gold codes of length $N=2^{5}-1$; (b) simulated signal $s(t, D)$ at a distance of propagation $D=200 \mathrm{~mm}$; matched filter response.

where $s_{w}(t)$ is the so-called warped signal, and $\dot{w}(f)$ represents the first derivative of $w(f)$. It has been shown in [17], that the discrete WFT can be efficiently computed as the composition of an inverse Fourier transform $\mathbf{F}^{-1}$ and a scaled non-uniform Fourier transform $\mathbf{F}_{N U}$ plus an anti-aliasing term which can be neglected in most applications:

$$
\mathbf{W}_{w} \simeq \mathbf{F}^{-1} \cdot \mathbf{F}_{N U}
$$

It is also worth noting that the WFT is a unitary time-frequency transformation that preserves the orthogonality of the transformed signals. In [16] it was shown that the WFT can be exploited to compensate a Lamb wave signal from the dispersion induced by the traveled distance. To this aim, the warping map $w(f)$ has to be defined through its functional inverse, such as:

$$
K \frac{d w^{-1}(f)}{d f}=\frac{1}{c_{g}^{n}(f)}
$$

where $K$ is a normalization parameter selected so that $w^{-1}(0.5)=w(0.5)=0.5$. Thanks to Eq. (4), the right 

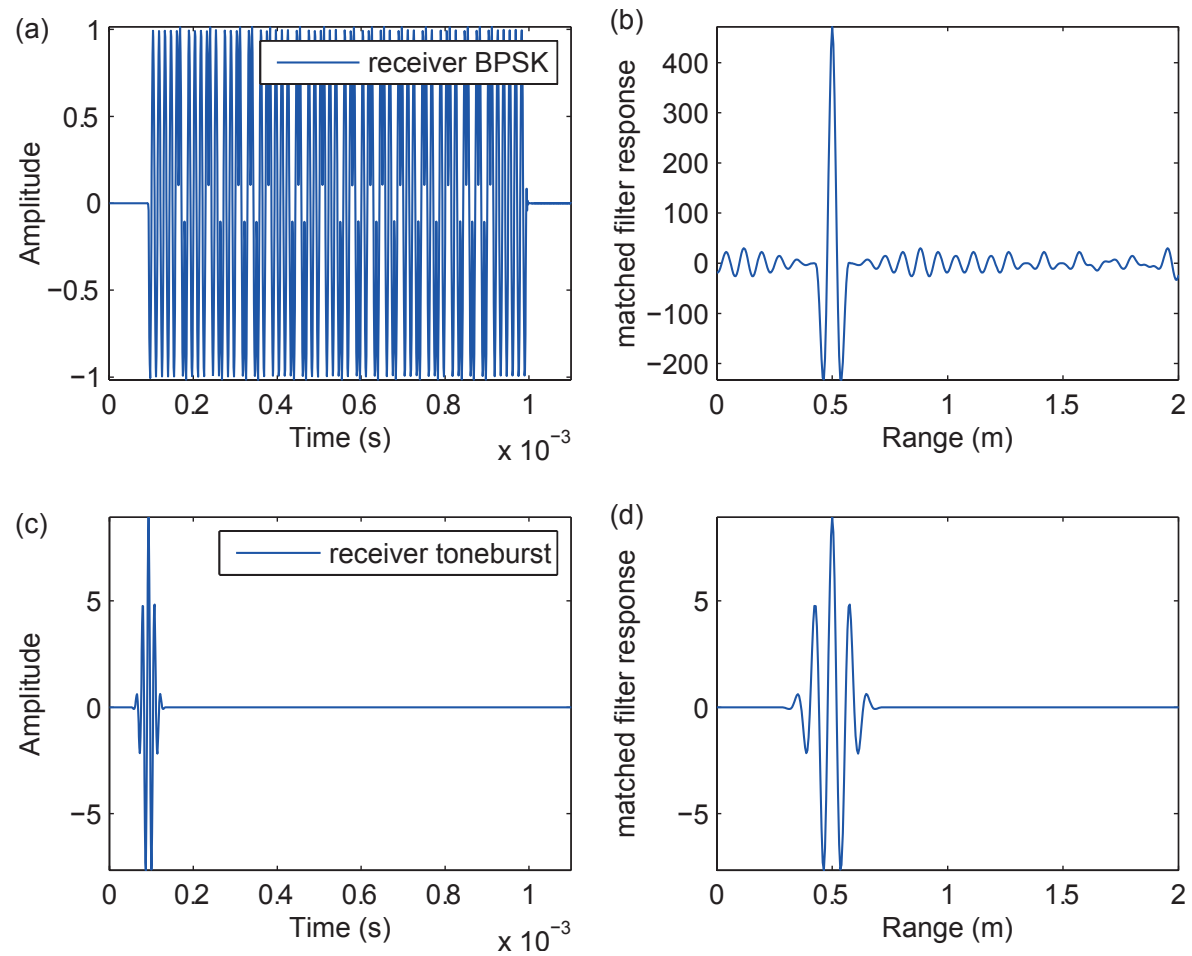

Figure 3: Analytical simulation example based on the fundamental symmetric mode (S0-mode): (a) receiver signal after a propagation distance of $0.5 \mathrm{~m}$; (b) corresponding matched filter response; (c) receiver signal of a standard toneburst excitation with 3 cycles after the same propagation distance; (d) corresponding matched filter response. In both cases the correct propagation distance is found, but the waveforms differ in terms of waveform extend as well as the sidelobes in the BPSK waveform.

hand term of Eq. (1) can be rewritten as:

$$
S(f, D)=S(f, 0) \cdot e^{-j 2 \pi D \int_{0}^{f} \frac{1}{c_{g}^{n}(\alpha)} d \alpha}=S(f, 0) \cdot e^{-j 2 \pi w^{-1}(f) K D}
$$

where the frequency distortion results from the nonlinear phase term $-j 2 \pi w^{-1}(f) K D$. Now by applying the warping operator to $s(t, D)$ we obtain a warped signal $s_{w}(t, D)=\mathbf{W}_{w}\{s(t, D)\}$ whose frequency transform is:

$$
S_{w}(f, D)=\mathbf{F W}_{w}\{s(t, D)\}=[\sqrt{\dot{w}(f)} S(w(f), 0)] \cdot e^{-j 2 \pi f K D}
$$

By looking at this equation, it can be clearly seen how the dispersive effect due to the traveled distance is converted into a simple time delay proportional to the distance itself $\tau_{D}^{w, n}=K D$, as the phase term presents a linear dependence on warped frequencies (i.e. frequencies of the warped signal).

\subsection{Warped cross-correlation}

This property can be fruitfully exploited by using signal correlation techniques, in fact the Fourier transform of the cross-correlation $x_{w}(T x, R x)$ between the warped acquired signal $s_{w}(t, D)$ and the warped actuated pulse $s_{w}(t, 0)$ is given by:

$$
\mathbf{F} x_{w}[T x, R x]=\mathbf{F W}_{w}\{s(t, D)\} \cdot \mathbf{F} \mathbf{W}_{w}^{*}\{s(t, 0)\}=\dot{w}(f) \cdot|S(w(f), 0)|^{2} \cdot e^{-j 2 \pi f K D}
$$


It follows that the abscissa value at which the envelope of the cross-correlation in Eq. (7) peaks can be directly related to the distance of propagation $D$. The cross-correlation of the warped version of the signals shown in Fig. 2(a) and (b) is depicted in Fig. 4. By comparing Fig. 2(c) and Fig. 4 it can be seen how beneficial is the proposed warped cross-correlation since it perfectly peaks for the actual distance traveled by the wave $D=200 \mathrm{~mm}$.

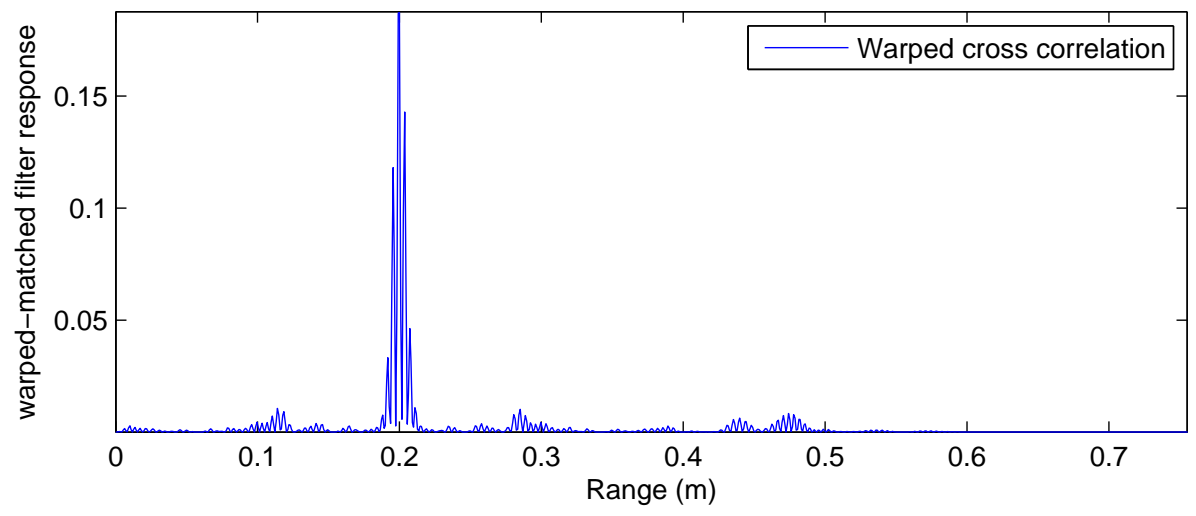

Figure 4: Warped cross-correlation between the signals depicted in Fig. 2(a) and (b). The waveform peaks in correspondence to the actual propagation distance.

A second numerical experiment is performed by considering two transmitters and one receiver. The first transmitter TX1 is located $200 \mathrm{~mm}$ far from the receiver RX, while the second transmitter TX2 is placed at a distance of $300 \mathrm{~mm}$ from RX. The actuation pulses at TX1 and TX2, i.e. $T x_{1}$ and $T x_{2}$, respectively, are sinusoids modulated by Gold codes and are depicted in Fig. 5(a) and (b), respectively. The signal received by RX, denoted as $R_{x}$, computed numerically considering the $A_{0}$ mode only propagating in a $3 \mathrm{~mm}$ thick aluminum plate, is a mixture of time-delayed, attenuated and dispersed versions of the two transmitted signals, and it is shown in Fig. 5(c).

The cross-correlation between the warped received signal $\mathbf{W}_{w}\{R x\}$ and the warped pulse at TX1 $\mathbf{W}_{w}\left\{T x_{1}\right\}$ is plotted in Fig. 5 (d), whereas the cross-correlation between the warped received signal and the warped pulse at TX2 $\mathbf{W}_{w}\left\{T x_{2}\right\}$ is shown in Fig. 5(e). As can be seen, the warped cross-correlation acts as a pulse compression and demodulation operator: the related waveforms peak at around $200 \mathrm{~mm}$ and $300 \mathrm{~mm}$ (see the two subplots 5(d) and (e), respectively), and have a very low intensity out of the neighborhood of the actual traveled distances, as desired. Therefore, such waveforms would be perfectly suited for wave traveling distance estimation procedures in the context of Lamb wave inspections systems based on "multiple TX".

Despite the different formalism, the dispersion compensation performed with the WFT is substantially analogous to the one presented in other papers $[18,19,20,21]$. However, the new formalism reveals the following noteworthy property:in force of Eq. (3) $\mathbf{W}_{w} \simeq \mathbf{F}^{-1} \cdot \mathbf{F}_{N U}$, therefore $\mathbf{F W}_{w}=\mathbf{F F}^{-1} \mathbf{F}_{N U}=\mathbf{F}_{N U}$, i.e. the warped cross-correlation can be rapidly computed by substituting the conventional Fourier Transform with a Non Uniform Fourier transform.

\subsection{Wavenumber filtering}

The final step of the proposed processing consists in applying the wavenumber filtering scheme presented in $[22,10][10]$ to the warped cross correlated signals $x_{w}[T x, R x]$ obtained by cross-correlating the warped version of the actuated signals and the received ones. 

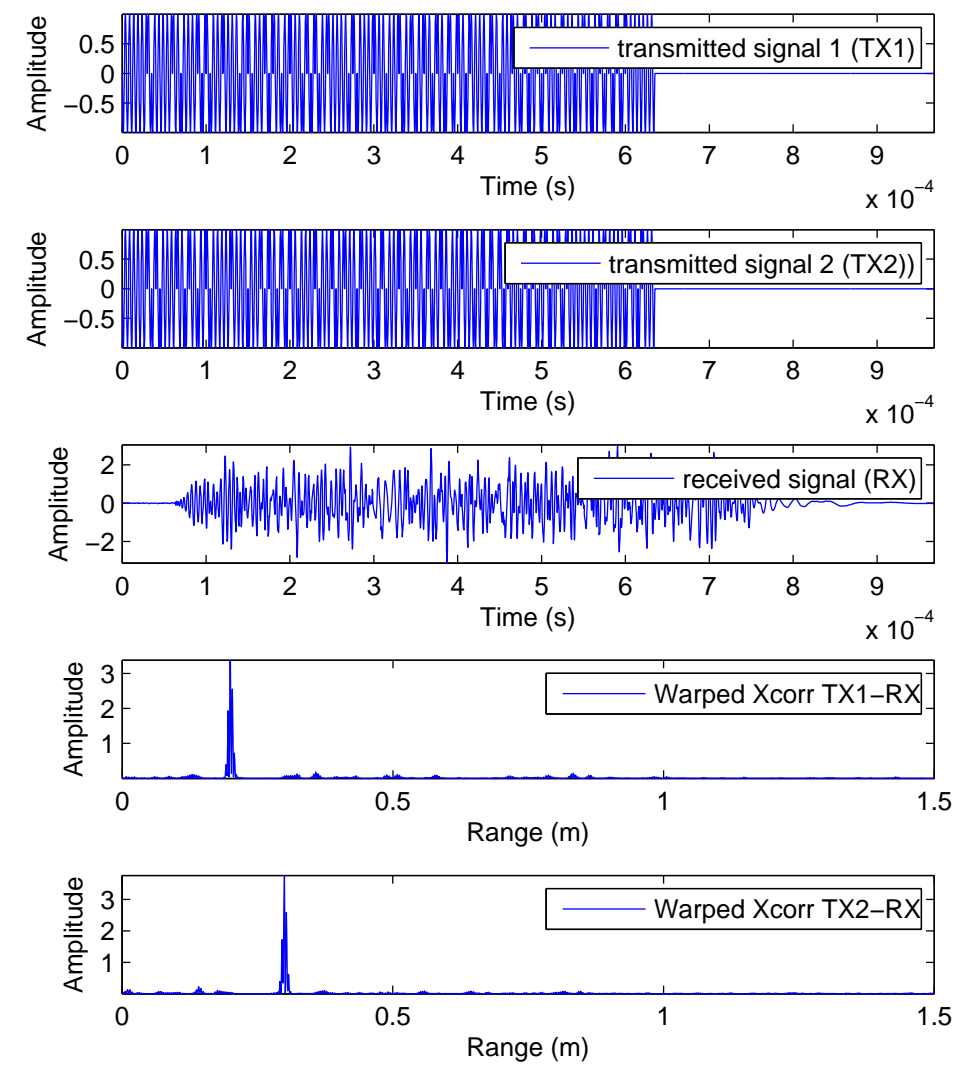

Figure 5: Numerical simulations of a setup constituted by two actuators (TX1 and TX2) and one sensor RX: (a) and (b) transmitted signals by TX1 and TX2, respectively; (c) received signal containing contributions from both transmitters; (d) warped cross-correlation between the signal transmitted by TX1 and the received signal; (e) warped cross correlation between the signal transmitted by TX2 and the received signal.

In particular, and in view of the experimental phase, the description here considers piezoelectric transducers as "multiple TXs" operating simultaneously on a plate-like component whereas a Laser Doppler Vibrometer capable to scan the entire plate-like surface is considered as waves detector. As such, multiple injected pulses related to the different actuators and the plate surface responses $s(x, y, t)$ at each point $x, y$ on the plate are available. The surface responses at each time instant $t=t^{*}$ form a wavefield image $s\left(X, Y, t^{*}\right)$ of the propagating elastic waves that have been actuated simultaneously by the transmitters $T X_{i}$ $(i=1,2,3, \ldots j)$.

Following the work given in [10], the proposed wavenumber filtering scheme utilizes 2D discrete Fourier transform of consecutive wavefield images $s\left(X, Y, t^{*}\right)$. The transform leads to wavenumber domain images. A filtering mask is applied in the wavenumber domain. Then, an inverse 2D Fourier transform is applied to each image. Such procedure produces an animation with amplified damage-induced effects. The last step of the algorithm consists in the calculation of the energy based root mean square value which takes 
into account the attenuation of elastic waves. Hence, the influence of damage is independent of the damage location.

Then, the full wavefield in the time domain $s(X, Y, t)$ is converted in multiple images $x_{w}\left[T X_{i}\right]\left(X, Y, n_{w}\right)$ in the warped domain (where $n_{w}$ are the warped time samples [16]). Such conversion is performed by eross-correlating the signals aequired at the different positions $x \in X, y \in Y$ with the pulse actuated by $i$ th actuator $\left(T X_{i}\right)$, as described in the previous subsection. It follows that, if the number of actuators is $j, j$ warped cross-correlated wavefields are available after this step.

In the following step, the wavenumber filtering mask has to be designed. Fo this aim, the warped eross-eorrelated signals are divided into two sets. The first set corresponds to the first $\mathrm{N}_{e}$ warped time samples of the excited pulse and the second set includes the remaining $N_{e}+1 \ldots N$ frames, where $N$ is the number of total samples of the warped cross-correlated signals. It is assumed that in proximity of the pulse actuator (i.e. the piezoelectric transducer) damage does not occur, so that there is no interaction of the wave front with any damage in the interval which corresponds to frames $1 \ldots N_{e}$. If this assumption is true, these $\mathrm{N}_{e}$ frames can be used as a digital fingerprint of the elastic waves propagating in the considered structure. More specifically, such fingerprint is computed by averaging the wavenumber domain representation of the selected warped cross-correlated wavefield images:

$$
X_{w}\left[T X_{i}\right]\left(k_{x}, k_{y}\right)_{A V G}=\frac{1}{N_{e}} \sum_{n_{w}=1}^{N_{e}} X_{w}\left[T X_{i}\right]\left(k_{x}, k_{y}, n_{w}\right)
$$

where $X_{w}\left[T X_{i}\right]\left(k_{x}, k_{y}, n_{w}\right)=\mathbf{F}_{2 D}\left[x_{w}\left[T X_{i}\right]\left(x, y, n_{w}\right)\right]$ is the 2D Fourier transform of $n_{w}$ th warped cross correlated wavefield image. Such transformation change the processed signal representation from space domain $(x, y)$ to wavenumber domain $\left(k_{x}, k_{y}\right)$. The implemented Wavenumber filtering is based on the idea that most of the wave propagation components matehing the digital fingerprint $X_{w}\left[T X_{i}\right]\left(k_{x}, k_{y}\right)_{A v G}$ are to be removed from the wave pattern. Consequently, the filter mask is computed as follows:

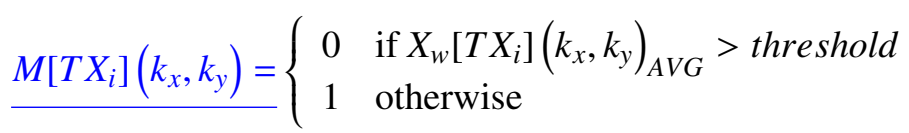

The threshold value is set so that about $5 \%$ elements of matrix $M$ are equal 0 . The wavenumber filtered signal is obtained by computing in wavenumber domain the product of the acquired warped cross-correlated wavefield image with the filter mask:

$$
\underline{\tilde{X}_{w}\left[T X_{i}\right]\left(k_{x}, k_{y}, n_{w}\right)=X_{w}\left[T X_{i}\right]\left(k_{x}, k_{y}, n_{w}\right) \cdot M\left[T X_{i}\right]\left(k_{x}, k_{y}\right)}
$$

It means that almost whole wave pattern will be removed in the first $N_{e}$ frames, whereas only the new wave components which correspond to anomalies (such as damage) will be preserved in the remaining frames.

where $\tilde{x}_{w}\left[T X_{i}\right]\left(x, y, n_{w}\right)=\mathbf{F}_{2 D}^{-1} \cdot \tilde{X}_{w}\left[T X_{i}\right]\left(k_{x}, k_{y}, n_{w}\right)$ is $2 \mathrm{D}$ inverse Fourier transform of $n_{w}$-th image resulting from the presented processing, and $v_{n w}$ is a weighting factor which is defined by taking into account wave attenuation and velocity (more details can be found in [10]).

\section{Experimental verification}

To validate the proposed methodology, an experiment using scanning laser vibrometry was performed. An aluminum plate $(1000 \times 1000 \times 5 \mathrm{~mm})$ was investigated. A cross-shaped notch was cut in the plate at 


\begin{tabular}{ccccc} 
& TX1 & TX2 & TX3 & TX4 \\
\hline$x(\mathrm{~mm})$ & 250 & 750 & 250 & 750 \\
\hline$y(\mathrm{~mm})$ & 750 & 750 & 250 & 250 \\
\hline
\end{tabular}

Table 1: Actuators topology

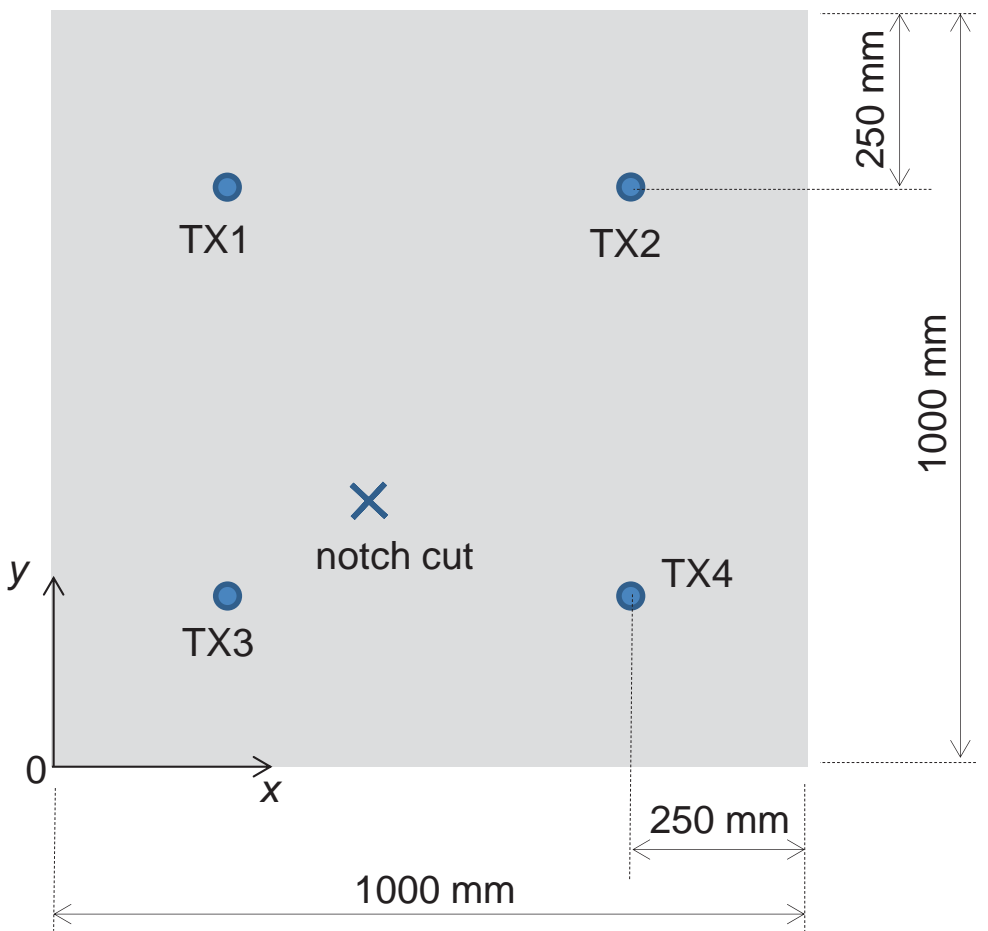

Figure 6: A sketch of the experimental set up used to validate the defect location procedure.

the location $(x=400 \mathrm{~mm}$ and $y=400 \mathrm{~mm})$. Four PZT discs (Ceramtec - Sonox P502, diameter $10 \mathrm{~mm})$ were bonded to the plate. The experiments were carried out by placing the transducers in the configuration reported in Table 1.

Figure 6 shows a sketch of the experimental set-up. Guided waves were excited by actuating simultaneously at the four PZT transducers pulses encoded with Gold codes of length $L=2^{7}-1$. A scanning Laser Doppler Vibrometer (SLDV) by Polytec was used to acquire the ultrasonic wave field generated over the whole plate. The SLDV sampling frequency $(1.28 \mathrm{MHz})$ is sufficiently high to avoid aliasing effects, as the frequency content of the acquired signal vanishes above $400 \mathrm{kHz}$.

Figure 7 shows the experimental wavefield detected at the time instants $t_{1}=46.5 \mu \mathrm{s}$ and $t_{2}=93 \mu \mathrm{s}$. As can be seen, in a short time it becomes difficult to distinguish the different wave component due to the long pulse duration and the simultaneous actuation performed. In addition, the defect presence cannot be noted from the acquired wavefield in the time domain.

In order to obtain the warped cross-correlated wavefields, first the WFT operator must be defined on the basis of the associated warping map. For such a scope, the Lamb waves group velocity dispersion curves for the 5-mm thick aluminum plate were calculated, as detailed in Sec. 3. In the [0-400] kHz frequency 
(a)

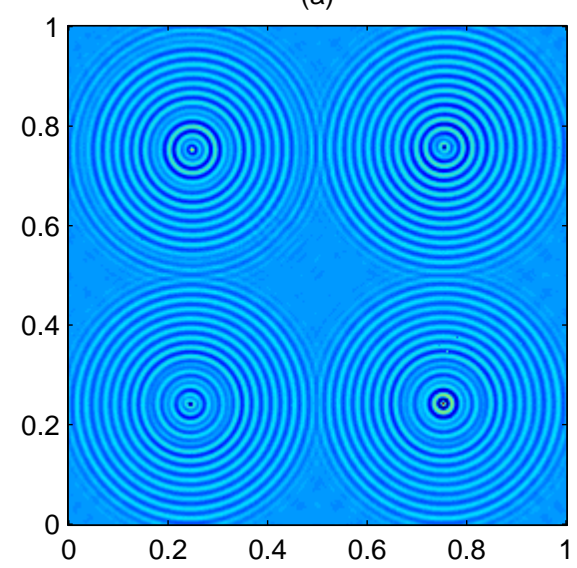

(b)

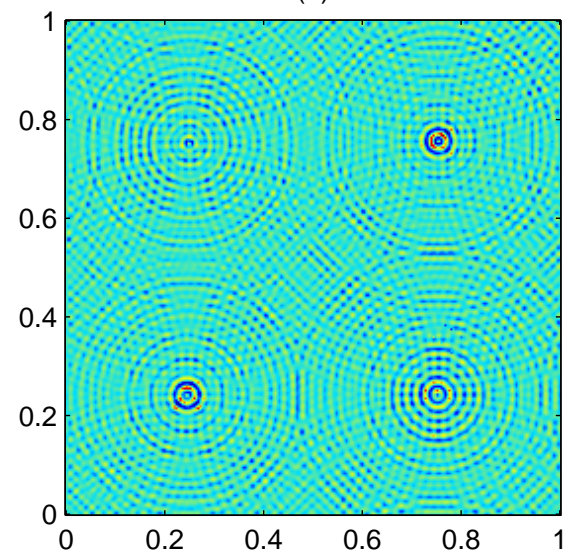

Figure 7: Snapshots of the wavefield acquired by the SLDV at the time instants $t_{1}=46.5 \mu \mathrm{s}(\mathrm{a})$, and $t_{2}=93 \mu \mathrm{s}(\mathrm{b})$.

range, only the two fundamental $A_{0}$ and $S_{0}$ waves can propagate through this plate. Since the energy in the $A_{0}$ mode is greater than the one retained by the $S_{0}$ mode, the group velocity curve of the $A_{0}$ mode was used to shape the warping map according to Eq. (4).

Next, the warped cross correlation between the acquired wavefield and the 4 different excited pulses were computed, i.e. $x_{w}\left[T X_{i}\right]\left(X, Y, n_{w}\right), i=1,2,3,4$. Thanks to this procedure, the group delay dependency on distance is removed. Four snapshots related to warped time sample $n_{w}=100$, corresponding to a distance of propagation equal to $220 \mathrm{~mm}$, are plotted in Fig. 8. As can be seen, the propagating waves are very effectively separated.

\subsection{Detailed wavefield analysis}

Fig. 9 shows a comparison of the wavefield for single firing (matched filter case), single firing (dispersion compensated case) and multiple firing (dispersion compensated case) at two different time instances. Comparing the two single firing scenarios shows that the effect related to dispersion can be neglected in the first time instance $(a, b)$, because of the short distance traveled by the wave. It is worth noting that in the second time instance the spreading caused by dispersion is now very evident $(\mathrm{d}, \mathrm{e})$. Dispersion compensation is now effective and leads to a significant pulse compression of the waveforms.

The single firing with dispersion compensation is the benchmark for the case of multiple simultaneous excitation shown in $(\mathrm{c}, \mathrm{f})$. Dispersion compensation is again effective in reducing the spreading of the waveforms. However, a lack of uniformity can be observed that are related to non-ideal cross-correlation properties of the Gold code sequences. This observation shows the need to develop novel waveforms that have superior cross-correlation properties. In addition, the small amplitude S0-mode excitation occurs four times now which also degrades the uniformity of the demodulated wavefield.

\subsection{Analysis of geometric beamspreading versus cross-correlation induced wavefield artifacts}

In order to further analyze the effect of dispersion and to provide a more quantitative insight into the pulse modulation and demodulation approach, Figure 5 shows the peak amplitude in the processed frames of the wavefield for three different scenarios. The normalization has been performed with respect to the 
cumulative signal energy of each frame of the wave field. The higher is the peak of the wavefield, the easier it is to distinguish the wavefront over the noise floor.

It can be observed that the red and blue curves show a similar decreasing trend which is related to the geometric beamspreading when the wave is propagating in the plate. The decreasing trend of the red curve is more rapid because of the additional spreading caused by the uncompensated dispersion. In the case of simultaneous firing we can see an additional effect, because the trend changes towards higher time samples. The reason therefore is given by the other interfering BPSK pulses coming from the other parallel excitations. This observation must be evaluated in future research in more detail.

\subsection{Damage visualization}

Finally, the defect imaging procedure was applied to the processed data. Such procedure operates on the wavenumber domain and it is very effective in accentuating interactions of elastic waves with cracks and hidden damages and simultaneously discarding reflections from the structural boundaries while operating with multiple TX. The result achieved by cascading the wavenumber filtering procedure with the warped pulse compression is depicted in Fig. 11 showing results for single and multiple simultaenous excitation. It can be seen in the figures that with the simultaneous excitation the cross-shape of the notch can be clearly identified, while this is not the case for single firing. In both cases the same time for data acquisition with the scanning Laser Doppler Vibrometer is used. This demonstrates the benefit of using multiple simultaneous excitations for damage detection and damage characterization. All four piezo-transducer may be easily pointed as well.In particular, the image was obtained by summing the 4 damage maps created by applying the wavenumber filtering procedure on the warped correlation between the acquired wavefield and the pulse emitted by each piezo-transducer separately.It is worth noting, how the cross-shaped notch is accurately imaged since not only its real position but also its exact shape and size can be identified.

\section{Conclusions}

In this work, a phase-modulation and pulse compression strategy for Lamb wave inspections which is based on Spread spectrum coding and the Warped Frequency transform was proposed. The implemented procedure is dedicated to multiple transmitter systems. The conventional approach is based on the serialization in time of the transmitting operation. An $N_{T}$-fold reduction in acquisition time can be achieved if $N_{T}$ transmitters are actuated simultaneously and the interference is canceled out thanks to the pulse decoding procedure. A specific benefit of coded excitation signals is given by its adaptive nature to increase the SNR of the received signals by simply increasing the code length of the excitation signal while maintaining quasi-orthogonal signal properties. This property results in a correlation gain that is beneficial for many guided wave based SHM applications. In addition, increasing the code length would increase the number of available codes that would allow a larger number of transducers to emit at the same time. Moreover, on a system level it is not required to have any switching hardware since the excitation signals are transmitted simultaneously. The damage imaging results that are based on the local wavenumber technique achieved superior imaging results for the multiple simultaenous excitation compared to a conventional single firing strategy. The damage imaging results achieved show that the usage of the WFT allows to remove the effects of dispersion and to increase the efficacy of pulse compression.

\section{Acknowledgement}

Pawel Kudela, Maciej Radzienski and Wieslaw Ostachowicz would like to thank Polish National Science Centre for their financial support in the frame of MAESTRO project entitled: Excitation and control of mechanical waves in nonlinear media (DEC-2013/10/A/ST8/00071). 


\section{References}

[1] Z. Su, L. Ye, Y. Lu, Guided lamb waves for identification of damage in composite structures: A review, Journal of sound and vibration 295 (3) (2006) 753-780.

[2] W. Staszewski, B. Lee, L. Mallet, F. Scarpa, Structural health monitoring using scanning laser vibrometry: I. lamb wave sensing, Smart Materials and Structures 13 (2) (2004) 251.

[3] M. Castaings, P. Cawley, The generation, propagation, and detection of lamb waves in plates using air-coupled ultrasonic transducers, The Journal of the Acoustical Society of America 100 (5) (1996) 3070-3077.

[4] S. Caporale, S. Callegari, M. Ricci, P. Burrascano, Excitations and signal processing for multiprobe setups, in: Ultrasonic Nondestructive Evaluation Systems, Springer, 2015, pp. 141-171.

[5] J. Moll, C.-P. Fritzen, Guided waves for autonomous online identification of structural defects under ambient temperature variations, Journal of Sound and Vibration 331 (20) (2012) 4587-4597.

[6] L. De Marchi, A. Perelli, A. Marzani, A signal processing approach to exploit chirp excitation in lamb wave defect detection and localization procedures, Mechanical Systems and Signal Processing 39 (1) (2013) 20-31.

[7] C. White, Data communications and computer networks: A business user's approach, Cengage Learning, 2010.

[8] A. J. Viterbi, CDMA: principles of spread spectrum communication, Addison Wesley Longman Publishing Co., Inc., 1995.

[9] L. De Marchi, A. Marzani, S. Caporale, N. Speciale, Ultrasonic guided-waves characterization with warped frequency transforms, Ultrasonics, Ferroelectrics, and Frequency Control, IEEE Transactions on 56 (10) (2009) 2232-2240.

[10] P. Kudela, M. Radzieński, W. Ostachowicz, Identification of cracks in thin-walled structures by means of wavenumber filtering, Mechanical Systems and Signal Processing 50 (2015) 456-466.

[11] B. Lee, E. S. Furgason, High-speed digital golay code flaw detection system, Ultrasonics 21 (4) (1983) 153-161.

[12] E. H. Dinan, B. Jabbari, Spreading codes for direct sequence cdma and wideband cdma cellular networks, Communications Magazine, IEEE 36 (9) (1998) 48-54.

[13] D. Hutchins, P. Burrascano, L. Davis, S. Laureti, M. Ricci, Coded waveforms for optimised air-coupled ultrasonic nondestructive evaluation, Ultrasonics 54 (7) (2014) 1745-1759.

[14] Moll, J., T. Wandowski, P. Malinowski, M. Radzienski, S. Opoka, W. Ostachowicz, Experimental Analysis and Prediction of Antisymmetric Wave Motion in a Tapered Anisotropic Waveguide, The Journal of the Acoustical Society of America, IF: 1.650138 (1) (2015) 299-306.

[15] I. Bartoli, A. Marzani, F. Lanza di Scalea, E. Viola, Modeling wave propagation in damped waveguides of arbitrary crosssection, Journal of Sound and Vibration 295 (2006) 685-707.

[16] L. De Marchi, A. Marzani, N. Speciale, E. Viola, A passive monitoring technique based on dispersion compensation to locate impacts in plate-like structures, Smart Materials and Structures 20 (2011) 035021-9pp.

[17] S. Caporale, L. De Marchi, N. Speciale, Fast computation of frequency warping transforms, IEEE Transactions on Signal Processing 58 (3) (2010) 1110-1121.

[18] A. Booer, J. Chambers, I. Mason, Fast numerical algorithm for the recompression of dispersed time signals, Electronics Letters 16 (13) (1977) 453-455.

[19] R. Sicard, J. Goyette, D. Zellouf, A numerical dispersion compensation technique for time recompression of Lamb wave signals, Ultrasonics 40 (1-8) (2002) 727-732.

[20] P. Wilcox, A rapid signal processing technique to remove the effect of dispersion from guided wave signals, IEEE Transactions on Ultrasonics, Ferroelectrics and Frequency Control 50 (4) (2003) 419-427.

[21] S. Fu, L. Shi, Y. Zhou, J. Cai, Dispersion compensation in lamb wave defect detection with step-pulse excitation and warped frequency transform, IEEE transactions on ultrasonics, ferroelectrics, and frequency control 61 (12) (2014) 2075-2088.

[22] E. B. Flynn, S. Y. Chong, G. J. Jarmer, J.-R. Lee, Structural imaging through local wavenumber estimation of guided waves, NDT \& E International 59 (2013) 1-10. doi:10.1016/j.ndteint.2013.04.003. URL http://linkinghub.elsevier.com/retrieve/pii/S0963869513000595 
(a)

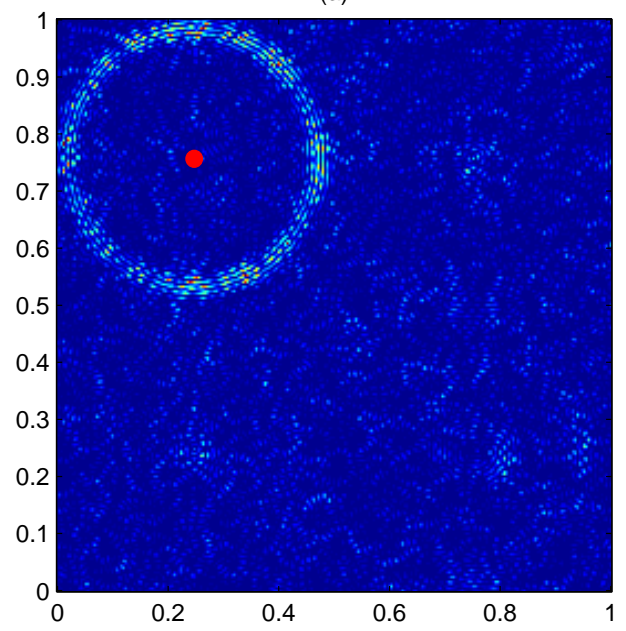

(c)

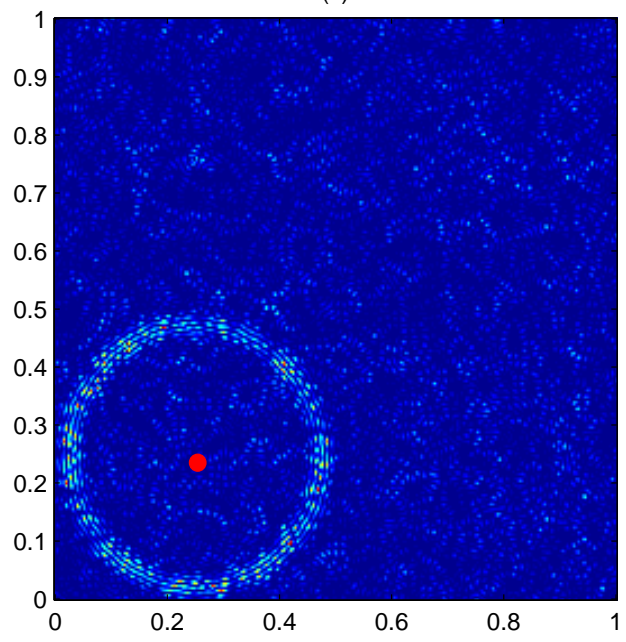

(b)

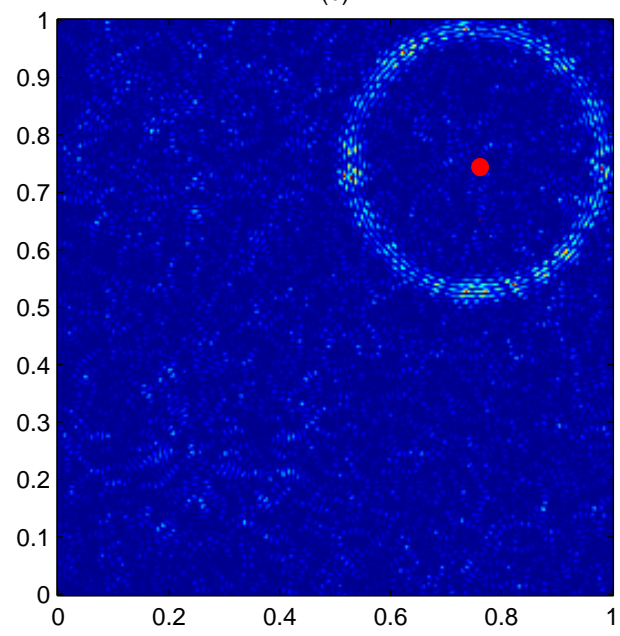

(d)

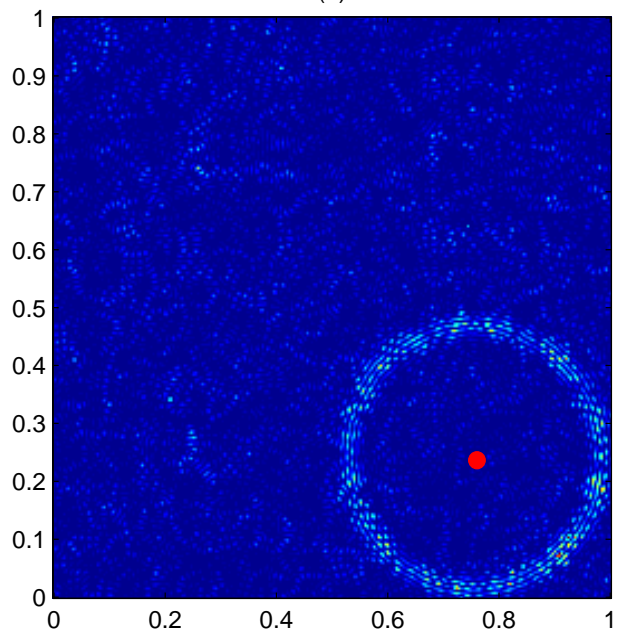

Figure 8: Wavefield demodulation achieved by performing the warped cross correlation between the acquired wavefield and the signals transmitted by TX1 (a), TX2 (b), TX3 (c) and TX4 (d), respectively. 


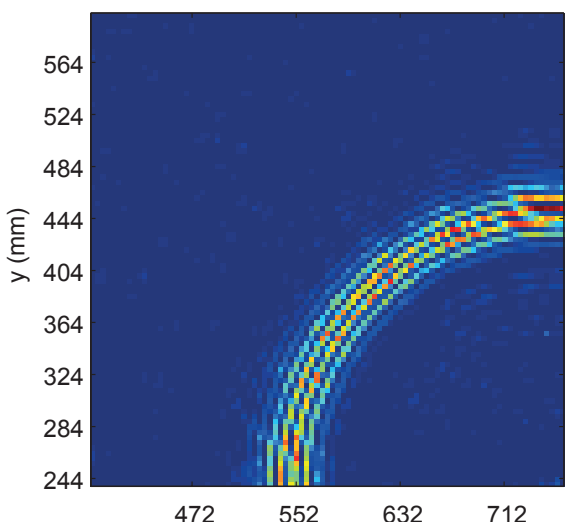

(a)

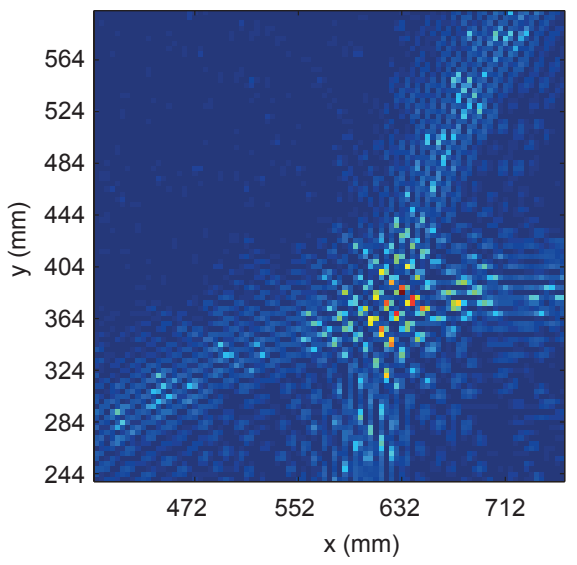

(d)

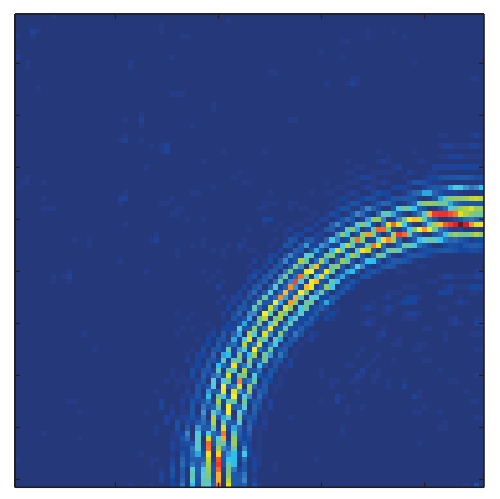

472

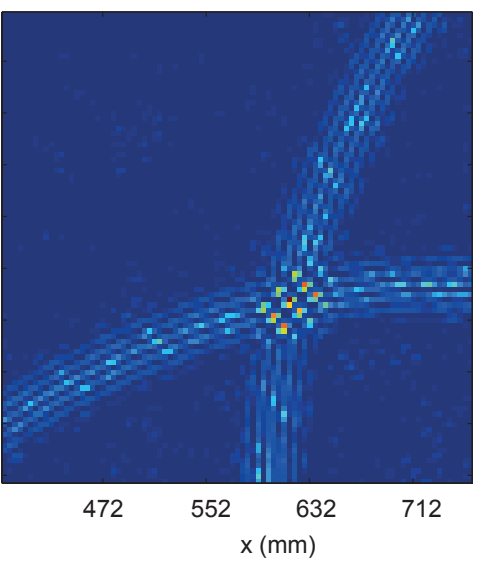

(e)

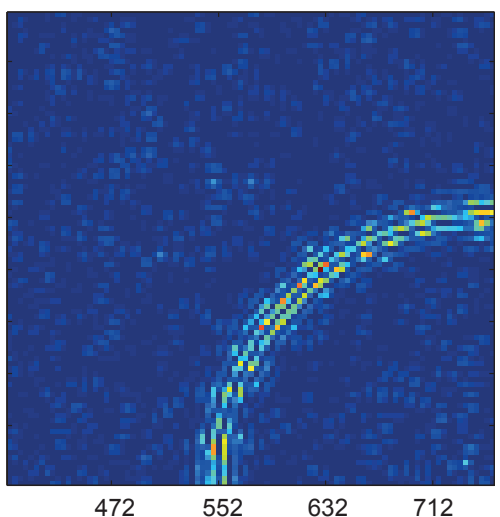

(c)

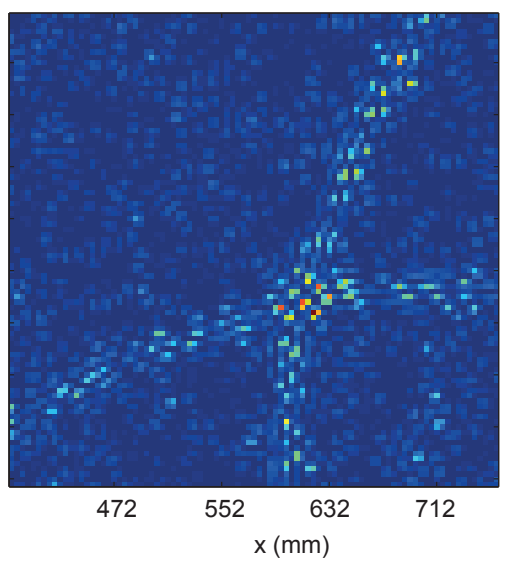

(f)

Figure 9: (First row) Snapshots of the wavefield at the beginning of the considered time interval: (a) is related to the single firing - matched filtering case; (b) is related to the single firing - dispersion compensated case; (c) is related to the multiple firing dispersion compensated case; (Second row) Snapshots of the wavefield captured at the end of the considered time interval: (d) is related to the single firing - matched filtering case; (e) is related to the single firing - dispersion compensated case; (f) is related to the multiple firing - dispersion compensated case. 


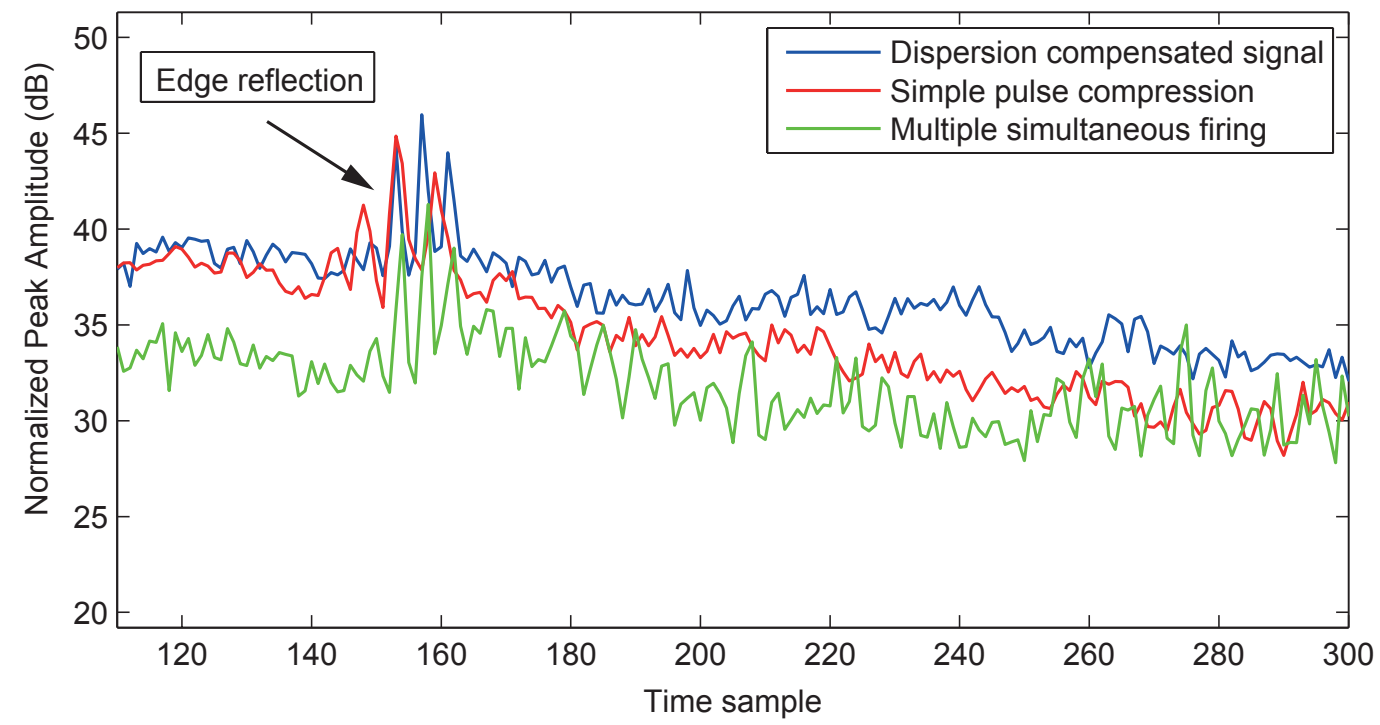

Figure 10: Normalized peak amplitude in the processed frames of the wavefield for the single firing (matched filtering case), single firing (dispersion compensated case) and multiple firing (dispersion compensated case).
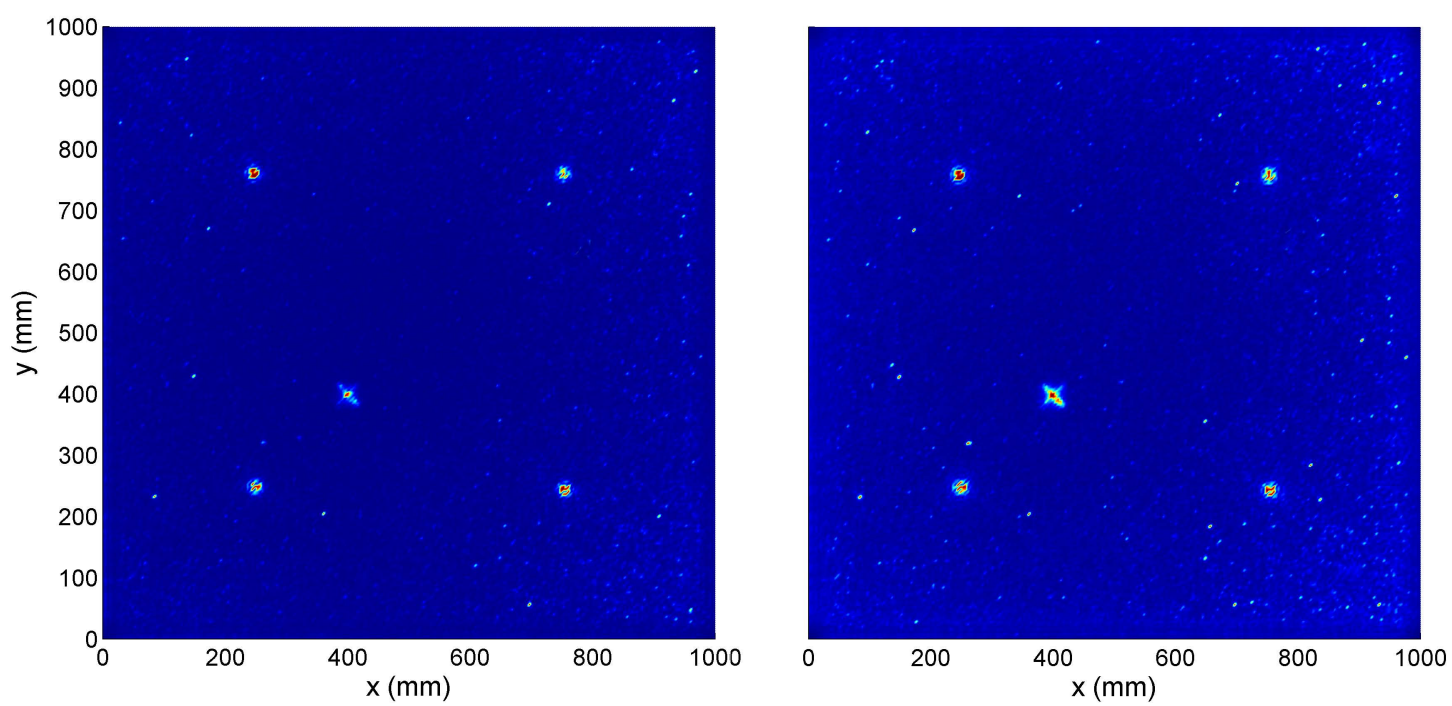

Figure 11: Damage imaging for (left) single excitation and (right) multiple simultaenous excitationĐamage imaging. 